\title{
Phascolarctobacterium faecium abundant colonization in human gastrointestinal tract
}

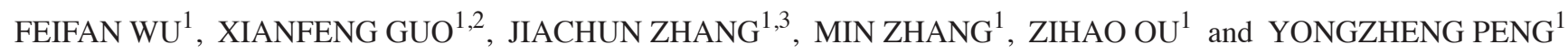 \\ ${ }^{1}$ Department of Laboratory Medicine, Zhujiang Hospital, Southern Medical University; \\ ${ }^{2}$ Department of Laboratory Medicine, Changsha Medical University; \\ ${ }^{3}$ Department of Clinical Laboratory, Shenzhen SheKou People's Hospital, Shenzhen, Guangdong 510282, P.R. China
}

Received March 6, 2017; Accepted July 21, 2017

DOI: $10.3892 / \mathrm{etm} .2017 .4878$

\begin{abstract}
Phascolarctobacterium can produce short-chain fatty acids, including acetate and propionate, and can be associated with the metabolic state and mood of the host. The present study investigated the colonization characteristics of Phascolarctobacterium faecium in healthy individuals $<1-80$ years old in Southern China. A total of 150 fresh fecal samples were collected, and bacterial DNA was isolated from these samples for quantitative polymerase chain reaction analysis. Phascolarctobacterium faecium demonstrated a high colonization rate and abundant colonization in the human gastrointestinal tract. The colonization rate varied between 43.33-93.33\%, and the abundance of Phascolarctobacterium faecium ranged between 3.22-5.76 log cells g-1 ( $<1$ years old) and 3.06-9.33 log cells g-1 ( $>1$ year old). The permillage of Phascolarctobacterium faecium in total bacteria ranged between 0.004-1.479. There was presence of Phascolarctobacterium faecium-like bacteria in younger individuals with a gradual increase in the number of bacteria maintained at a high level with increasing ages (between 1 and 60 years old), but with a decrease in elderly individuals ( $>60$ years old). The results of the present study demonstrated that Phascolarctobacterium faecium is abundantly colonized in the human gastrointestinal tract.
\end{abstract}

Correspondence to: $\mathrm{Dr}$ Yongzheng Peng, Department of Laboratory Medicine, Zhujiang Hospital, Southern Medical University, 253 Middle of Gongye Avenue, Guangzhou, Guangdong 510282, P.R. China

E-mail: yzpeng1981@126.com

Abbreviations: SCFA, short-chain fatty acids; $P$. faecium, Phascolarctobacterium faecium; OTUs, operational taxonomic units; CT, threshold cycle; PCR, polymerase chain reaction

Key words: Phascolarctobacterium faecium, real-time polymerase chain reaction, colonization rate, age-related distribution, gut microbiota

\section{Introduction}

The human gut microbiota is composed of 400-500 species of microbes. However, the molecular classification of operational taxonomic units (OTUs) indicate the presence of more than 1,000 OTUs (OTUs, equivalent to species) in the gut of each in different societies, and that the number of OTUs increases with age $(1,2)$. The gene pool of the microbial habitants of the gut is extremely diverse and considerably larger than the gene pool of the host, which determines a number of metabolic capacities of the bacteria that are essential for the survival of these organisms in the gut $(1,3,4)$.

In recent years, high throughput sequencing technologies have revealed the correlation between gut microbiota and the host. Phascolarctobacterium was found to be a substantial acetate/propionate-producer that could be dramatically increased by berberine and metformin. This in turn may contribute to the beneficial effects of the two drugs on the host (5). Moreover, Phascolarctobacterium was found to be positively correlated to the positive mood of the human (6). An increasing number of studies proposed that Phascolarctobacterium faecium (P.faecium) exerted beneficial effects on the host, including rat model of nonalcoholic fatty liver (7).

$P$.faecium, which utilizes succinate and produces propionate, was first purified from koala feces in 1992. P. faecium are obligate anaerobic, Gram-negative, non-spore-forming, non-motile, asaccharolytic, and belonging to firmicutes (8). Although uncultured colonies closely related to $P$. faecium were frequently detected in samples from the human gastrointestinal tract, isolation of Phascolarctobacterium from the human gastrointestinal tract and the expansion of the culture are not yet described in the literature (9), thereby limiting the functional studies and clinical applications. In addition, the gut microbiota can be affected by aging and diet habit. For example, the elderly subjects as compared to the adult population show a reduction in the diversity of the microbiota, characterized by a considerable interindividual variability, with lower numbers of firmicutes (10). The bacterial composition can also be affected by the diet. Human gut bacterial communities like $P$. faecium are altered by the addition of cruciferous vegetables to a regulated fruit- and vegetable-free 
diet (11). P. faecium may have undergone a widespread distribution in the human gastrointestinal tract. As the intestinal microbiota changes during aging, presumably, the level of intestinal $P$. faecium may be different during the human life. Thus, the detection of the presence of this bacterium requires an in-depth investigation.

Therefore, we have attempted to isolate $P$. faecium bacteria from human feces and investigated the distribution of $P$. faecium-like bacteria in human feces using real-time PCR analysis with species-specific primers.

\section{Materials and methods}

Isolation of strains of P. faecium. Fecal samples were freshly collected from healthy Chinese females (age, median 25 years old $)$ and serially diluted in pre-reduced PBS $(0.15 \mathrm{M}$ $\mathrm{NaCl}, 0.15 \mathrm{M}$ sodium phosphate, $0.05 \mathrm{M}$ phosphate-buffered saline; $\mathrm{pH} 7.0$ ) from $10^{-1}$ to $10^{-5} \mathrm{~g}$ and immediately transferred into an anaerobic glove box containing $80 \% \mathrm{~N}_{2}, 10 \% \mathrm{CO}_{2}$, and $10 \% \mathrm{H}_{2} .100 \mu \mathrm{l}$ fecal diluents were then inoculated to the chocolate plate (Detgerm Microbiology Technology, Guangzhou, China) and incubated at $37^{\circ} \mathrm{C}$ for 5 days. Single pinpoint colonies were selected and regrown on chocolate plates. This step was repeated until pure cultures were obtained. Finally, two strains have been achieved with pure cultures. 16S rDNA from each was PCR amplified using the universal primers 27f(59-AGAGTTTGATCCTGGCT-39) and 1492r(59-GGTTACCTTGTTACGACTT-39) (12). The $16 \mathrm{~S}$ rDNA gene sequences of the strains were submitted to GenBank, and similar sequences were searched for in the public databases using the BLAST algorithm. The sequence similarity was analyzed with the EzTaxon-e-server (13). MEGA5.0 software was used to construct the phylogenetic tree by the neighbor-joining method $(14,15)$.

Fecal sample collection. Fecal samples from healthy infant subjects aged $<1$ year old $(n=30), 1-10$ years old $(n=30)$, 10-30 years old $(n=30), 30-60$ years old $(n=30)$, and $60-80$ years old $(n=30)$, were collected and DNA extracted as described below. All the individuals we tested were in southern China, who consumed high proportion of carbohydrate and a moderate amount of fat, protein and vegetables daily in general.

DNA extraction from human fecal samples. Total DNA was extracted from the fecal samples by a QIAamp Fast DNA Stool Mini kit (Hilden, Germany), following the manufacturer's instructions.

Species-specific primer design. Two specific primers were designed from the variable regions of the 16S RNA gene sequence of $P$. faecium. The GenBank program of NCBI (BLAST) showed that both the primers were specific to the P. faecium. The primers PF1 (5'GGCGGCTTAATAAGTCGA GC3') and PF2 (5'CGTTCGCTACCCTGGCTTTC3') amplified a 200 bp amplicon.

Primers for examining the species specificity. Primers were purchased from Sangon Biotech Co., Ltd (Shanghai, China). The specificity was further confirmed by the DNA

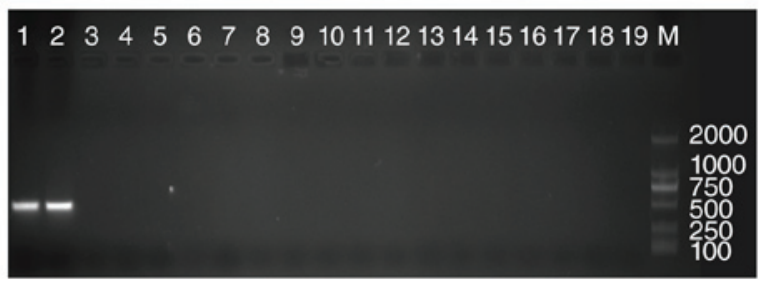

Figure 1. P. faecium specific primer confirmation by PCR. Lanes 1 and 2 were clinically-isolated $P$. faecium (strain 1 and strain 2, respectively), lane 3: Propionibacterium, lane 4: Micrococcus, lane 5:Nocardia, lane 6:Bacteroides, lane 7: Flavobacterium, lane 8: Sphingobacterium, lane 9: Bacillus, lane 10: Staphylococcus, lane 11: Enterococcus, lane 12: Lactobacillus, lane 13: Clostridium, lane 14: Listeria, lane 15: Neisseriales, lane 16: Burkholderia, lane 17: Salmonella, lane 18: Pseudomonas, lane 19: negative control, lane M: marker.

extracted from different species, comprising four phyla: (1) Actinobacteria: 2 species of Propionibacterium, 3 of Micrococcus and 2 of Nocardia. (2) Bacteroidetes: 5 Bacteroides, 2 Flavobacterium and 3 Sphingobacterium. (3) Firmicutes: 10 Bacillus, 5 Staphylococcus, 6 Enterococcus, 8 Lactobacillus, 4 Clostridium and 2 Listeria. (4) Proteobacteria: 2 Neisseriales, 8 Burkholderia, 10 Salmonella, 1 Pseudomonas. These cultures were provided by The Clinical Laboratory Culture Collection of Zhujiang Hospital. The DNA extract of the above strains was utilized in real-time PCR. The results did not reveal any amplified signal from all these strains. DNA electrophoresis post-PCR substantiate the specificity of the primers (Fig. 1).

Real-time PCR on P.faecium. The real-time PCR standard curve of $P$. faecium was built using $\mathrm{SYBR}^{\circledR}$ Select Master Mix (\#44729, Life Technologies, Grand Island, NY, USA), following the manufacturer's protocol, in an ABI vii7 real-time PCR System (Applied Biosystems, USA). The DNA samples were pre-denatured at $95^{\circ} \mathrm{C}$ for 2 min (first stage) and then amplified with 40 cycles at $95^{\circ} \mathrm{C}$ for $15 \mathrm{sec}$, at $58^{\circ} \mathrm{C}$ for $30 \mathrm{sec}$, at $72^{\circ} \mathrm{C}$ for $60 \mathrm{sec}$, and the final extension at $72^{\circ} \mathrm{C}$ for $5 \mathrm{~min}$. The fluorescent product was detected in the last step of each cycle. To distinguish the target PCR product from the non-target product, melting curve analysis was performed after amplification using the default settings of the ABI software from 60 to $95^{\circ} \mathrm{C}$.

The standard curve was created using 10-fold serial dilutions of $P$. faecium pure culture DNA corresponding to $10^{3}$ to $10^{9}$ cells/reaction, determined microscopically by DAPI staining method (16). The bacterial concentration of each sample was estimated by comparing the threshold cycle (CT) values obtained from the standard curve. All the samples were analyzed in triplicate. A linear correlation between the cell counts and the CT values ( $2=0.998$ ) was observed when the number of cells/reaction mixture was between $10^{3}$ and $10^{9}$. The melting curve analysis of the amplicons obtained by real-time PCR generated a distinct peak at $82^{\circ} \mathrm{C}$ in the $16 \mathrm{~S}$ rRNA gene of P. faecium.

Statistical analysis. The data were expressed as median with interquartile ranges in the parentheses. Man-Whitney U test was used to estimate the amount of $P$. faecium in feces, 
Table I. No. of P. faecium-like bacteria/g of feces and percentage in total bacteria

\begin{tabular}{lcccc}
\hline $\begin{array}{l}\text { Group } \\
\text { (years) }\end{array}$ & $\begin{array}{c}\text { No. of } P \text {. faecium } \\
\text { colonized sample/total } \\
\text { No. of sample }\end{array}$ & $\begin{array}{c}\text { log no. of } \\
\text { P. faecium } \\
\text { cells/g feces }\left(^{\mathrm{a}}\right)\end{array}$ & $\begin{array}{c}\text { log no. of total } \\
\text { bacteria cells/g } \\
\text { feces }\left(^{\mathrm{a}}\right)\end{array}$ & $\begin{array}{c}\text { \% relative to } \\
\text { the total } \\
\text { no. of cells }\end{array}$ \\
\hline$<1$ & $13 / 30(43.33 \%)$ & $3.78(3.22-5.76)^{\mathrm{b}}$ & $9.14(8.47-10.92)$ & 0.004 \\
1 to 10 & $21 / 30(70 \%)$ & $7.30(3.06-9.33)$ & $10.32(9.46-10.91)$ & 0.955 \\
10 to 30 & $27 / 30(90 \%)$ & $7.55(4.20-9.26)$ & $10.64(10.35-10.93)$ & 0.812 \\
30 to 60 & $28 / 30(93.33 \%)$ & $7.68(3.86-9.03)$ & $10.51(10.16-10.76)$ & 1.479 \\
$>60$ & $27 / 30(90 \%)$ & $6.82(3.18-9.37)$ & $10.56(9.722-10.96)$ & 0.195 \\
\hline
\end{tabular}

No., number. ${ }^{a}$ Due to a non-normal distribution, microbial data were expressed as median, with interquartile ranges in parentheses. ${ }^{b}$ significant differences $(\mathrm{P}<0.01)$ in fecal samples were determined between age $<1 \mathrm{y}$ and the other different age stages, by Man-Whitney U test.

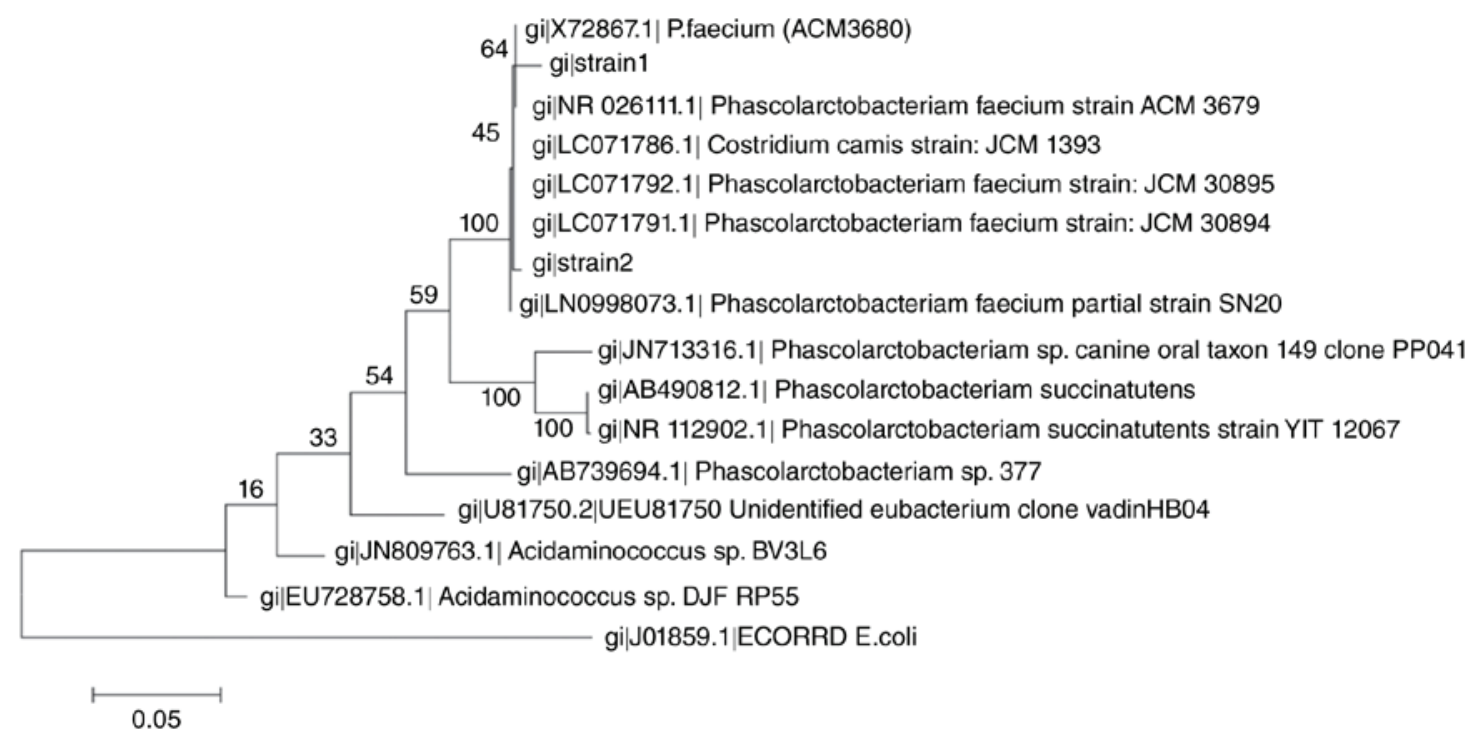

Figure 2. Phylogenetic tree showing the position of the two clinically isolated P. faecium strains (strain1 and strain2) among the selected clones or strains belonging to Phascolarctobacterium. The tree, which was rooted using Escherichia coli as the outgroup, was generated by the neighbor-joining method.

whereas the $\chi^{2}$ test was used to analyze the colonization rate in various stages of age $(\mathrm{P}<0.05$ was considered to indicate a statistically significant difference). All data were analyzed by SPSS20.0 software (IBM SPSS, Armonk, NY, USA).

\section{Results}

Successful isolation of P. faecium from human feces. $P$. faecium was successfully isolated from the human gut. The $16 \mathrm{~S}$ rDNA sequence indicated that the strain belonged to the originally-isolated strain from koala feces, with $99 \%$ similarity. The phylogenetic tree showed that the position of these two clinically isolated strains among the selected clones or strains belonged to Phascolarctobacterium (Fig. 2).

P. faecium have a high colonization rate in gastrointestinal tract. As presented in Fig. 3, a P. faecium colonization characteristic analysis was conducted. The colonization rate was calculated by the number of $P$. faecium colonized samples/total number of samples in the same age stage. Five different age stages presented different colonization rates.
The highest colonization rate in the five age stages was found in individuals aged 30-60 years old (93.33\%). Individuals $<1$ year old showed the lowest colonization rate (43.33\%). The colonization rate increased quickly from infant to youth (70\%), 1-10 years old. Adults aged 10-30 years old exhibited a similar colonization rate as compared to the elder group $(90 \%)$. The statistical results by $\chi^{2}$ test were represented in Fig. $3 \mathrm{C}$. The colonization rate in infants is significantly low to those aged 10-30 years old, 30-60 year-old adults, and elders $(\mathrm{P}<0.01)$. On the other hand, youth aged 1-10y also exhibits a significantly low colonization rate as compared to adults aged 30-60y $(\mathrm{P}<0.05)$ (Fig. 3C).

P. faecium abundantly colonized the gastrointestinal tract. The values ranged from 3.22-5.76 log cells $\mathrm{g}^{-1}$ and 3.06-9.33 log cells $\mathrm{g}^{-1}$ in samples from infants (age $\left.<1 \mathrm{y}\right)$ to youth $(1 \mathrm{y}<\mathrm{age}<10 \mathrm{y})$. The highest level was presented in adults aged between 30-60y, ranging from 3.86-9.03 log cells $\mathrm{g}^{-1}$. Adults aged from 10-30y and elders reveal values, 4.20-9.26 and 3.18-9.37, respectively. Remarkably, the concentration of $P$. faecium cells in the fecal samples from infants 
A

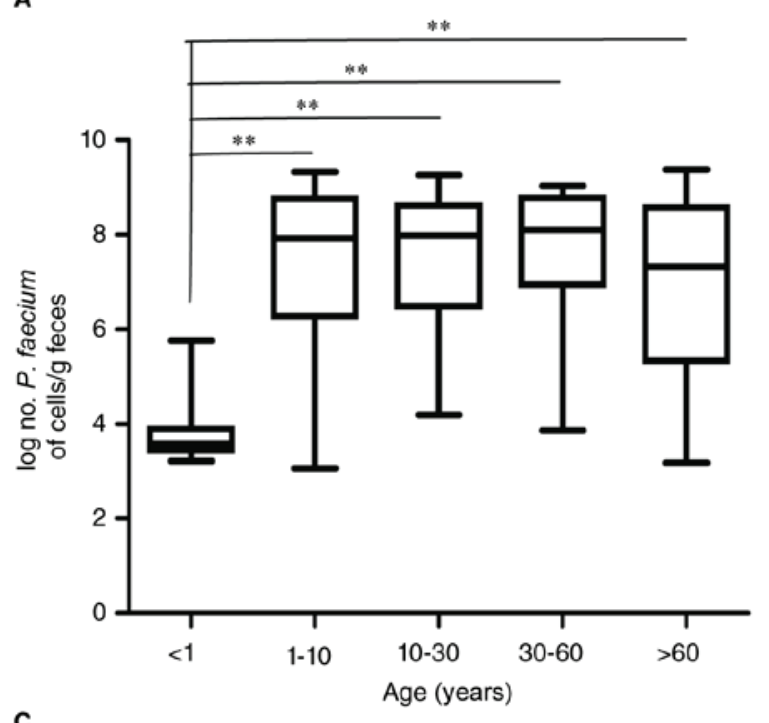

C

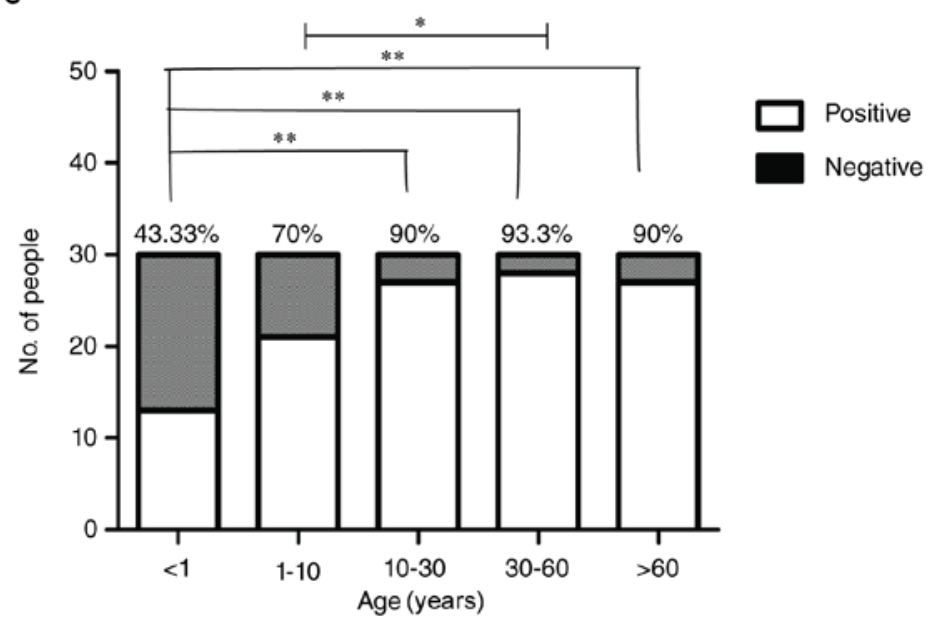

B

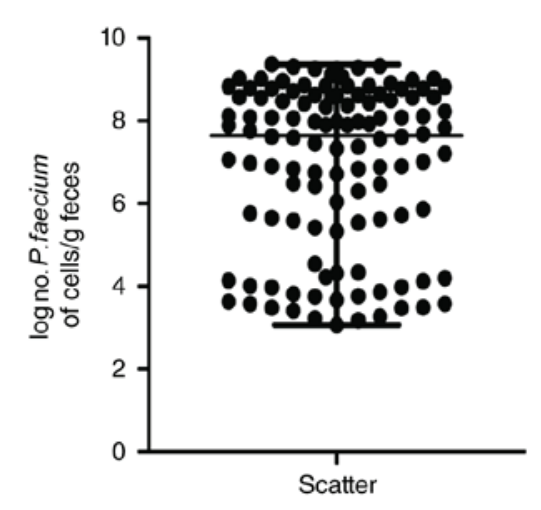

D

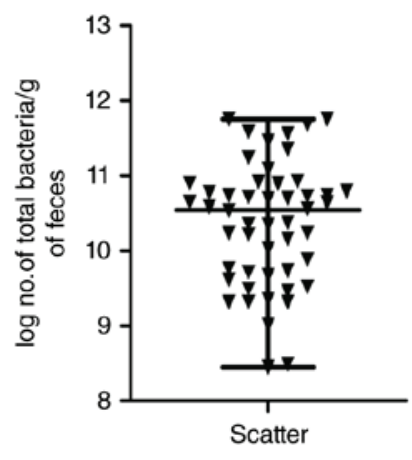

Figure 3. P. faecium colonization characteristic analysis. (A) Log no. P. faecium of cells $\mathrm{g}^{-1}$ feces from five different age stages; significant differences were determined by $\chi^{2}$ test. (B) The scatter spot distribution of $\log$ no. $P$. faecium of cells $\mathrm{g}^{-1}$ feces. (C) No. of positive and negative individuals and colonization rate in five different age stages; significant differences were determined by Mann-Whitney $\mathrm{U}$ test. (D) The scatter spot distribution of log No. of total bacteria $\mathrm{g}^{-1}$. Microbial data were expressed as median, with interquartile ranges in parentheses as non-normal distribution. ${ }^{*}$ means significant differences $\mathrm{P}<0.05^{* *}$ means significant differences $\mathrm{P}<0.01$.

was significantly less $(\mathrm{P}<0.01)$ as compared to the other sample groups determined by a Mann-Whitney U test (Table I, Fig. 3A and $\mathrm{B})$.

P. faecium-like bacteria were present at a high level in total bacterial population. Total bacteria in samples ranged from 9.14-10.64 log cells $\mathrm{g}^{-1}$ in the fecal samples (Table I, Fig. 3D). The relative abundance of $P$. faecium in total bacteria (\%o) was calculated by the amount of $P$. faecium/ the amount of total bacteria*1000. Each sample was calculated separately and their means in each group were presented in Table I. The permillage of $P$. faecium in total bacteria ranged from $0.004-1.479$. This data revealed a high colonization status of $P$. faecium in the gastrointestinal tract (Table I).

Age distribution of $P$. faecium-like bacteria. P. faecium-like bacteria appeared in early-life and increased in adult stage followed by a decrease in the elderly age. According to the colonization rate, value, and permillage of total bacteria, $P$. faecium-like bacteria appeared in early-life, increased in adult age, and then subsequently decreased in the elder age. This was a common bacteria colonized in the gastrointestinal tract.

\section{Discussion}

The lumen of the human gastronintestinal tract contains trillions of commensal bacteria, which are difficult to cultivate. $P$. faecium was first purified from koala feces. Since then, additional reports of $P$. faecium have been lacking. We firstly isolated some strains of $P$. faecium from the human gastrointestinal tract. The isolation of the genus of Phascolarctobacterium from human feces was a herculean task as a tip-size colony had to be harvested from Columbia Blood Agar plate after approximately one week.

Culture-dependent techniques, which are a beneficial way to elucidate the gut bacterial repertoire, can be used only for a limited range of bacteria in the human gut (17). Thus, improving the culture-dependent methods for the isolation of $P$. faecium from feces remains a key challenge in microbiology. 
In the current study, we found that this species not only has a high colonization rate but is also abundantly colonized in the human gastrointestinal tract. This may suggest its critical role in the dynamic balance of the gut microbiota. The colonization rate ranged from 43.33 to $93.33 \%$ and the amounts ranged from 3.22-5.76 log cells $\mathrm{g}^{-1}$ and 3.06-9.33 log cells $\mathrm{g}^{-1}$, according to different age stages. This confirmed that aging was a crucial factor for the dynamic balance of the gut flora. Age distribution showed that P. faecium-like bacteria were present in the early-life, and the number increased rapidly in people aged 1-60 years old, the number decreased in elders (>60 years old). This phenomenon may be attributed to the diet habit. A previous study demonstrated that rats fed with high-fat diet appeared to have a higher amount of the short chain fatty acid producers, including P.faecium (18). Besides, Phascolarctobacterium spp. specialise in the utilisation of succinate produced by other bacteria (8). In parallel, the abundance of Bacteroides and Parabacteroides, both major producers of succinate, increased by high fat diet and was positively correlated with body weight (19). The elderly and individuals aged less than 1 year consumed relative less fat and have a relative low body weight, this may resulted in Bacteroides and Parabacteroides decreased, and the available succinate for P. faecium was decreased. Physical exercise was another factor accounting for Phascolarctobacterium decrease in the elderly. Rats with a low capacity for running that were fed an acute high-fat diet appeared to decrease the amount of Phascolarctobacterium and be more susceptible to nonalcoholic fatty liver disease (7). Compared with the young, the elderly population have less physical exercise, which may be another reason for Phascolarctobacterium decrease.

Of note, individuals in these two age stages $(<1$ year and $>60$ years old, respectively) are more susceptible to digestive and metabolic diseases, which are likely linked to the less amount of $P$. faecium. $P$. faecium-like bacteria are also present at a high percentage in total bacteria. The permillage of $P$. faecium in total bacteria ranged from 0.004 to 1.479 . In addition, $P$. faecium was firstly isolated from koala, an animal fed on poisonous eucalyptus leaves, it may have link with the detoxication of gut microbiota. Thus, $P$. faecium may exert a beneficial role in the human gastrointestinal tract. However, further investigation is imperative to delve into the functional role of these strains in the human gastrointestinal tract.

Herein, we demonstrate, for the first time, the corroboration and development of a quantitative real-time PCR for the detection and quantification of bacteria related to $P$. faecium. Our results demonstrate that $P$. faecium is present and colonizes the gastrointestinal tract in early-life and develops to a high level in healthy adults followed by a decrease in elders. Further studies are a prerequisite to exploring the role of $P$. faecium in the microbiota development and its role in human health.

\section{Acknowledgments}

The present study was supported by grants from the National Natural Science Foundation of China (No. 81441066), the Natural Science Foundation of Guangdong Province (S2013010014850), the Guangdong Provincial Department of
Science and Technology (2015A020213002), and Guangzhou Kangze Medical Science and Technology Co., Ltd fund (201511).

\section{References}

1. Ramakrishna BS: Role of the gut microbiota in human nutrition and metabolism. J Gastroenterol Hepatol 28 (Suppl 4): S9-S17, 2013.

2. Yatsunenko T, Rey FE, Manary MJ, Trehan I, Dominguez-Bello MG, Contreras M, Magris M, Hidalgo G, Baldassano RN, Anokhin AP, et al: Human gut microbiome viewed across age and geography. Nature 486: 222-227, 2012.

3. Shanahan F: The colonic microbiota in health and disease. Curr Opin Gastroenterol 29: 49-54, 2013.

4. Goodman AL and Gordon JI: Our unindicted coconspirators: Human metabolism from a microbial perspective. Cell Metab 12: 111-116, 2010.

5. Zhang X, Zhao Y, Xu J, Xue Z, Zhang M, Pang X, Zhang X and Zhao L: Modulation of gut microbiota by berberine and metformin during the treatment of high-fat diet-induced obesity in rats. Sci Rep 5: 14405, 2015.

6. Li L, Su Q, Xie B, Duan L, Zhao W, Hu D, Wu R and Liu H: Gut microbes in correlation with mood: Case study in a closed experimental human life support system. Neurogastroenterol Motil 28: 1233-1240, 2016.

7. Panasevich MR, Morris EM, Chintapalli SV, Wankhade UD, Shankar K, Britton SL, Koch LG, Thyfault JP and Rector RS: Gut microbiota are linked to increased susceptibility to hepatic steatosis in low-aerobic-capacity rats fed an acute high-fat diet. Am J Physiol Gastrointest Liver Physiol 311: G166-G179, 2016.

8. Dot TD, Osawa $R$ and Stackebrandt E: Phascolarctobacterium faecium gen. nov, spec. nov., a Novel Taxon of the Sporomusa Group of Bacteria. Syst Appl Microbiol 16: 380-384, 1993.

9. Watanabe Y, Nagai F and Morotomi M: Characterization of Phascolarctobacterium succinatutens sp. nov., an asaccharolytic, succinate-utilizing bacterium isolated from human feces. Appl Environ Microbiol 78: 511-518, 2012.

10. Rondanelli M, Giacosa A, Faliva MA, Perna S, Allieri F and Castellazzi AM: Review on microbiota and effectiveness of probiotics use in older. World J Clin Cases 3: 156-162, 2015.

11. Li F, Hullar MAJ, Schwarz Y and Lampe JW: Human gut bacterial communities are altered by addition of cruciferous vegetables to a controlled fruit- and vegetable-free diet. J Nutr 139: 1685-1691, 2009.

12. Jordan EM, Thompson FL, Zhang XH, Li Y, Vancanneyt M, Kroppenstedt RM, Priest FG and Austin B: Sneathiella chinensis gen. nov., sp. nov., a novel marine alphaproteobacterium isolated from coastal sediment in Qingdao, China. Int J Syst Evol Microbiol 57: 114-121, 2007.

13. Kim OS, Cho YJ, Lee K, Yoon SH, Kim M, Na H, Park SC, Jeon YS, Lee JH, Yi H, et al: Introducing EzTaxon-e: A prokaryotic $16 \mathrm{~S}$ rRNA gene sequence database with phylotypes that represent uncultured species. Int J Syst Evol Microbiol 62: 716-721, 2012.

14. Saitou N and Nei M: The neighbor-joining method: A new method for reconstructing phylogenetic trees. Mol Biol Evol 4: 406-425, 1987.

15. Tamura K, Peterson D, Peterson N, Stecher G, Nei M and Kumar S: MEGA5: Molecular evolutionary genetics analysis using maximum likelihood, evolutionary distance, and maximum parsimony methods. Mol Biol Evol 28: 2731-2739, 2011.

16. Morikawa K and Yanagida M: Visualization of individual DNA molecules in solution by light microscopy: DAPI staining method. J Biochem 89: 693-696, 1981.

17. Eckburg PB, Bik EM, Bernstein CN, Purdom E, Dethlefsen L, Sargent M, Gill SR, Nelson KE and Relman DA: Diversity of the human intestinal microbial flora. Science 308: 1635-1638, 2005.

18. Lecomte V, Kaakoush NO, Maloney CA, Raipuria M, Huinao KD, Mitchell HM and Morris MJ: Changes in gut microbiota in rats fed a high fat diet correlate with obesity-associated metabolic parameters. PLoS One 10: e126931, 2015.

19. Lecomte V, Kaakoush NO, Maloney CA, Raipuria M, Huinao KD, Mitchell HM and Morris MJ: Changes in gut microbiota in rats fed a high fat diet correlate with obesity-associated metabolic parameters. PLoS One 10: e126931, 2015. 